\title{
Composite photocathodes for visible photon imaging with gaseous photomultipliers
}

\author{
E. Shefer", A. Breskin, A. Buzulutskov ${ }^{\mathrm{a}}, \mathrm{R}$. Chechik and M. Prager ${ }^{\mathrm{b}}$ \\ Department of Particle physics, The Weizmann Institute of Science, 76100 Rehovot, 1srael.
}

\begin{abstract}
We report on the results of coating K-Cs-Sb visible photocathodes, with thin $\mathrm{CsBr}$ and $\mathrm{CsI}$ protective films, in view of their possible combination with gas electron multipliers. Absolute quantum efficiency, photoelectron transport through the coating film, the effect of exposure to oxygen and to air and the photocathode behavior under intense photon flux are presented. The coated $\mathrm{K}-\mathrm{Cs}-\mathrm{Sb}$ photocathodes have typically maximum quantum efficiency values of 5-6\% at $312 \mathrm{~nm}$. They can withstand exposure to 150 Torr of oxygen for more than half an hour. Appropriate electron multipliers and possible applications are briefly discussed.
\end{abstract}

Presented at the Wire Chamber Conference

Vienna, February 22-26, 1998.

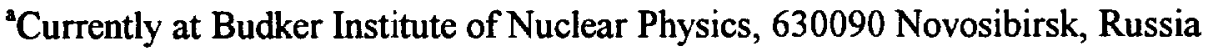

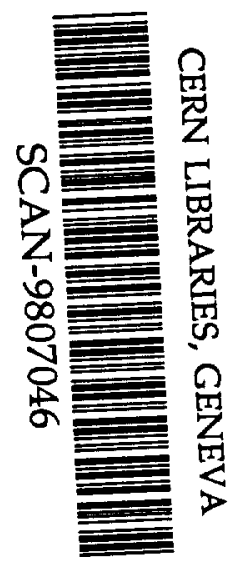

${ }^{b}$ ELAM Ltd. Jerusalem, Israel

"corresponding author: fnefrat@wis.weizmann.ac.il 


\section{Introduction}

Photon detectors with solid photocathodes coupled to gaseous electron multipliers [1,2] are attractive devices. They have large gain, sensitivity to single photoelectrons, fast response and, compared to vacuum photomultipliers, can be made very large and be less sensitive to magnetic fields. CsI-based detectors have been successfully applied for X-ray imaging at Synchrotron Radiation Sources [3] and for UV-photon imaging [4-6] in the field of Cherenkov Ring Imaging (RICH) devices. It is desirable to extend their spectral sensitivity range towards the visible light where numerous potential applications exist.

Alkali-antimonide photocathodes, highly efficient in the visible range, are extremely reactive to impurities in counting gases. Their operation in gas avalanche mode could be made possible by coating them with thin protective dielectric films $[7,8]$. Such protection is achieved at the expense of a reduction in quantum efficiency (QE). Systematic studies [9-12] have shown that it is possible to obtain a good protection capability and reasonable $Q E$, by a careful optimization of the coating material and its thickness for each type of photocathode. Furthermore, UV-sensitive coating materials, extend the composite visible photocathode sensitivity, to the UV.

We have extensively investigated coating of $\mathrm{Cs}_{3} \mathrm{Sb}$ and $\mathrm{K}-\mathrm{Cs}-\mathrm{Sb}$ photocathodes by alkali-halides, oxides and organic films [9-12]. We report here on our best results, achieved with $\mathrm{K}-\mathrm{Cs}-\mathrm{Sb}$ photocathodes coated with $\mathrm{CsBr}$ and $\mathrm{CsI}$ films.

\section{Results}

The experimental setup, developed for the preparation and protection of bialkali-antimonide photocathodes, and for in-situ investigations of their properties, is discussed in ref. [13].

The attenuation of the QE at $312 \mathrm{~nm}$ as a function of the film thickness for CsBr- and CsI-coated photocathodes is shown in fig.1. For coating thickness greater than $50 \AA$ the QE decay is exponential, representing the photoelectron attenuation in the coating film. Films of a few hundred angstroms thickness were chosen in order to obtain stability in oxygen environment while maintaining a reasonable QE. Typical quantum efficiency spectra of reflective bare and coated K-Cs-Sb photocathodes are shown in fig. 2. The bare photocathode has a QE of approximately $30 \%$ at $250-330 \mathrm{~nm}$. Coating it with $250-$ $300 \AA$ thick films results in a decrease of the QE; the $300 \AA \mathrm{CsBr}$-coated photocathode has typically $\mathrm{QE}$ values superior to $5 \%$ at $290-320 \mathrm{~nm}$ with a peak of $5.5 \%$ at $312 \mathrm{~nm}$, and the $250 \AA$ CsI-coated photocathode has QE values above $4 \%$ at $250-325 \mathrm{~nm}$, with a peak of $5 \%$ at $312 \mathrm{~nm}$. Our best $\mathrm{CsBr}-$ coated K-Cs-Sb photocathodes, have QE values reaching $8.5 \%$ at $312 \mathrm{~nm}$. Absolute QE spectra of CsI and $\mathrm{CsBr}$, highly efficient in the UV range $[5,14]$, are also shown in fig.2. The coated $\mathrm{K}-\mathrm{Cs}-\mathrm{Sb}$ photocathodes are therefore expected to have a sensitivity also in the UV.

The coating results in a dramatic increase of the photocathode stability under exposure to oxygen. Figure 3 shows the evolution of the absolute QE of bare and coated $\mathrm{Cs}_{3} \mathrm{Sb}$ and $\mathrm{K}-\mathrm{Cs}$-Sb photocathodes as a function of the residual oxygen pressure inside the chamber; each data point represents 5 minutes of exposure. The bare photocathodes totally degrade after a 5 minutes exposure to $10^{-5}$ Torr of oxygen; while the CsI- and CsBr- coated K-Cs-Sb photocathodes remain stable even when exposed to 150Torr, which is the partial pressure of oxygen in air. $\mathrm{CsBr}$-coated $\mathrm{Cs}_{3} \mathrm{Sb}$ displays similar stability in oxygen but a lower QE, and CsI- and $\mathrm{NaI}-$ coated $\mathrm{Cs}_{3} \mathrm{Sb}$ photocathodes have lower $\mathrm{QE}$ and poorer stability. The $\mathrm{QE}$ of CsI-coated K-Cs-Sb photocathodes remains practically unchanged even after a 1.5 hours exposure to 150Torr of oxygen, as shown in fig.4. Protection against impurities in counting gases at the ppm level, might require thinner protection layers, resulting in higher residual QE of the photocathodes (see fig.2). This is the subject of current investigations. 
The coated photocathodes decay more rapidly in contact with air. The $\mathrm{CsBr}$-coated $\mathrm{K}-\mathrm{Cs}-\mathrm{Sb}$ photocathode was stable only up to 5 minutes of exposure to 0.5 Torr of ambient air. We suspect that the damage is caused by moisture, which modifies the surface of the coating film. It was recently observed [16] that uniformly evaporated $\mathrm{CsBr}$ films, form clusters after short exposure to moisten argon. Thin CsI films were found to be less vulnerable, and therefore are expected to better protect the photocathodes.

Coating the photocathodes alters their physical and electrical properties, requiring detailed studies of their characteristics under photon illumination and gas multiplication. The stability of $\mathrm{CsBr}$-coated photocathodes was investigated. Surface upcharging was measured in $1 \mathrm{~atm}$. of methane at wavelength of $254 \mathrm{~nm}$ with photon flux between $2 \times 10^{9}$ and $3 \times 10^{10}$ photons $/ \mathrm{sec} \mathrm{mm}^{2}$; the results are shown in fig. 5 . For photon flux of $2 \times 10^{9}$ photons $/ \mathrm{sec} \mathrm{mm}^{2}$, practically no upcharging was observed. For the unrealistically large flux of $3 \times 10^{10}$ photons $/ \mathrm{sec} \mathrm{mm}^{2}$, a decrease of $10 \%$ in the photocurrent was measured in the first 5 seconds, followed by a decrease of another $15 \%$ over half an hour. The photocurrent recovered within 15 minutes after stopping the illumination. No upcharging was observed for bare K-Cs-Sb photocathodes, even at the highest photon flux.

Photocathodes aging by photon impact was measured under the same conditions, at wavelengths between 254 and $633 \mathrm{~nm}$ and is not expected to prevent the use of such photocathodes in detectors. Figure 6 shows the aging at a photon flux of $3 \times 10^{10}$ photons $/ \mathrm{sec} \mathrm{mm}$, as a function of the number of accumulated photons per $\mathrm{mm}^{2}$. A QE decrease by $50 \%$ was measured after accumulating $6 \times 10^{15}$ photons $/ \mathrm{mm}^{2}$. No clear dependence of the aging on the photon flux or wavelength was observed. To evaluate this degradation one could think of a critical application like mammography or radiography requiring very high instantaneous $\mathrm{X}$-ray flux. For instance, with a scintillator coupled to a photocathode and a gaseous multiplier, the QE will decay by $10 \%$ after $10^{14}$ photons $/ \mathrm{mm}^{2}$, equivalent to about $10^{5}$ radiographic images. It is interesting to note that the $\mathrm{QE}$ of the bare photocathode remained unchanged after accumulating $3 \times 10^{15}$ photons $/ \mathrm{mm}^{2}$. The mechanism responsible for the aging of coated photocathodes is being investigated. It may be related to ion displacement resulting from the ionic conduction of alkali-halides, to lattice distortion due to electron accumulation in the coating film and its interface with the photocathode, or to a photolysis effect similar to that observed for CsI $[5,17]$.

Our current experimental setup does not permit photoelectron multiplication in-situ, preventing measurements of photocathode aging due to ion impact. However, gas multiplication of photoelectrons emitted from a $\mathrm{CsBr}$ photocathode deposited on stainless steel substrate, measured in a parallel-plate mode, resulted in stable operation at a charge gain of $10^{4}$, under photon flux exceeding $10^{5}$ photons $/ \mathrm{sec}$ $\mathrm{mm}^{2}$. Therefore, the deposition of the dielectric $\mathrm{CsBr}$ film on the photocathode should not affect the detector operation under high gains, as already demonstrated with gas detectors operating with CsI photocathodes [6].

\section{Conclusions}

We have shown that $\mathrm{K}-\mathrm{Cs}-\mathrm{Sb}$ reflective photocathodes, coated with thin $\mathrm{CsBr}$ and $\mathrm{CsI}$ films have QE values exceeding $5 \%$, (in some cases reaching $8.5 \%$ ), at $312 \mathrm{~nm}$. These photocathodes are very stable in oxygen environment. In particular, the CsI-coated photocathode can withstand exposure to 150Torr of oxygen for 1.5 hours. Upcharging and aging by intense photon flux are not expected to limit the stable operation of detectors incorporating such photocathodes. The photocathodes are expected to have an extended sensitivity range from UV to visible. This can be very attractive for some applications, e.g. in fast $\mathrm{RICH}$ detectors and for the simultaneous reading of fast and slow photon components of scintillatiors e.g. that of $\mathrm{BaF}_{2}$. Detectors with such photocathodes could already find potential applications in numerous fields, such as $\mathrm{RICH}$, scintillation calorimetry and scintillation fiber tracking, 
medical imaging etc. An interesting application of protected photocathodes could be in the field of lasertriggered intense electron sources for RF guns [18], where currently employed alkali-antimonide and other photocathodes rapidly decay under inadequate vacuum conditions.

The present levels of stability of coated photocathodes in oxygen and moisture permit photocathode handling in glove box allowing for simple handling and detector assembly.

Minimizing the flux of back-drifting avalanche ions, in order to decrease possible damage by ion sputtering, is an important aspect in choosing the electron multiplication geometry. Gaseous electron multipliers with micro-patterned electrodes e.g. of microstrip, microgap, and microdot geometries are considered. In such multipliers a large fraction of the avalanche ions is collected on the electrode, limiting the photocathode damage [19]. Inserting a Gaseous Electron Multiplier (GEM) between the photocathode and the electron multiplier could also decrease ion impact as well as reduce the photon feedback [20].

The work was partially supported by the Israeli Science Foundation. A.Breskin is the W.P.Reuther professor of research in the peaceful use of atomic energy. 


\section{References}

[1] J.Edmends et al. Nucl. Instr. and Meth. A273 (1988) 145.

[2] G.Charpak et al. IEEE Trans. Nucl. Sci. 30 (1983) 134.

[3] R.Chechik et al. IEEE Trans. Nucl. Sci. NS-43 (1996) 1248.

[4] A.Breskin Nucl. Instr. and Meth. A367 (1995) 326 and references therein.

[5] A.Breskin Nucl. Instr. and Meth. A371 (1996) 116 and references therein.

[6] F. Piuz Nucl. Instr. and Meth. A371 (1996) 96 and references therein.

[7] V.Peskov et al. Nucl. Instr. and Meth. A353 (1994) 184.

[8] R.Enomoto et al. Nucl. Instr. and Meth. A343 (1994) 117.

[9] A.Breskin et al. Appl. Phys. Lett. 69 (1996) 1008.

[10] A.Buzulutskov et al. Nucl. Instr. and Meth. A387 (1997) 176.

[11] A.Buzulutskov et al. Nucl. Instr. and Meth. A400 (1997) 173.

[12] A.Breskin et al. Removable organic protective coating for alkali-antimonide photocathodes, preprint WIS-97/38/Dec.-PP, submitted to Nucl. Instr. and Meth. A.

[13] E.Shefer et al. Laboratory production of efficient alkali-antimonide photocathodes, preprint WIS-97/40/Dec.-PP, Nucl. Instr. and Meth. A, in press.

[14] E.A.Taft et al. J Phys. Chem. Solids 3 (1957) 1.

[15] E.Shefer et al. CsBr UV photocathodes, in preparation.

[16] T.Boutboul et al. Ultra-violet photoabsorption measurements in alkali-iodide and cesium-bromide evaporated films, preprint WIS-97/41/Dec.-PP, J. Appl. Phys, in press.

[17] J.Vavra et al. Nucl. Instr. and Meth. A387 (1997) 154.

[18] A.di Bona et al. Nucl. Instr. and Meth. A385 (1997) 385.

[19] E.Shefer et al. in: Proc. Int. Workshop on Micro-Strip Gas Chambers, Legnaro, Oct. 13-14 1994, p.107.

[20] R.Chechik et al. First results on the GEM operated at low gas pressure, proceedings of this conference.

\section{Figure captions}

Fig.1: The attenuation of the absolute quantum efficiency of $\mathrm{CsBr}$ - and CsI-coated $\mathrm{K}-\mathrm{Cs}-\mathrm{Sb}$ photocathodes as a function of the coating film thickness, at $312 \mathrm{~nm}$; the lines are exponential fits to the data.

Fig.2: Typical absolute QE spectra of K-Cs-Sb photocathodes, bare and coated with $300 \AA$ thick $\mathrm{CsBr}$ and $250 \AA$ thick CsI films and of CsI [5] and CsBr [15] photocathodes. All photocathodes are reflective. Fig.3: The results of photocathode exposure to oxygen. Shown is the evolution of the absolute QE values of bare and coated photocathodes as a function of the residual oxygen pressure measured at $312 \mathrm{~nm}$; the type and coating thickness are given in the figure. Exposure time at each data point is 5 minutes, followed by a QE measurement in vacuum.

Fig.4: Absolute quantum efficiency spectra of K-Cs-Sb photocathode coated with $250 \AA$ of CsI, unexposed and exposed to 150 Torr of oxygen for 5,25 and 85 minutes.

Fig.5: Upcharging of a K-Cs-Sb photocathode coated with $300 \AA$ of $\mathrm{CsBr}$, in $1 \mathrm{~atm}$. $\mathrm{CH}_{4}$. Shown is the relative photocurrent of the photocathode as a function of exposure time to photon flux of 2,8 and $30 \times 10^{9}$ photons $/ \mathrm{sec} \mathrm{mm}^{2}$.

Fig.6: Aging by photons in a current collection mode (with no gain) at $254 \mathrm{~nm}$, under photon flux of $3 \times 10^{10}$ photons $/ \mathrm{sec} \mathrm{mm}{ }^{2}$ in $1 \mathrm{~atm}$. $\mathrm{CH}_{4}$, of a K-Cs-Sb photocathode coated by $300 \AA$ thick $\mathrm{CsBr}$ film, as a function of the total number of accumulated photons per $\mathrm{mm}^{2}$. 


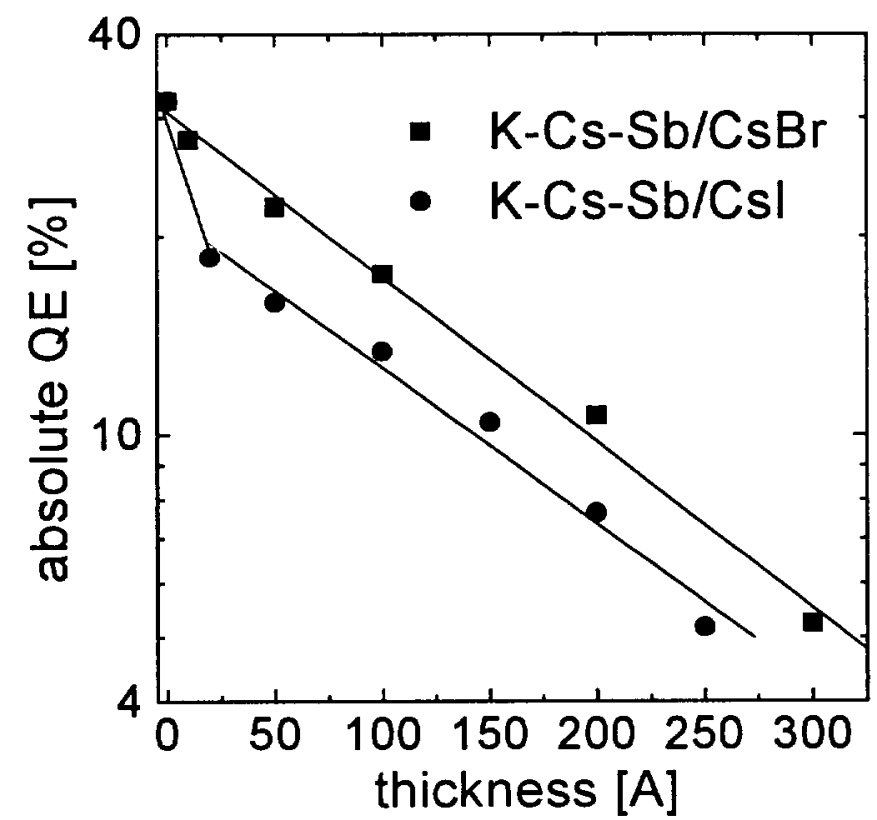

Figure 1

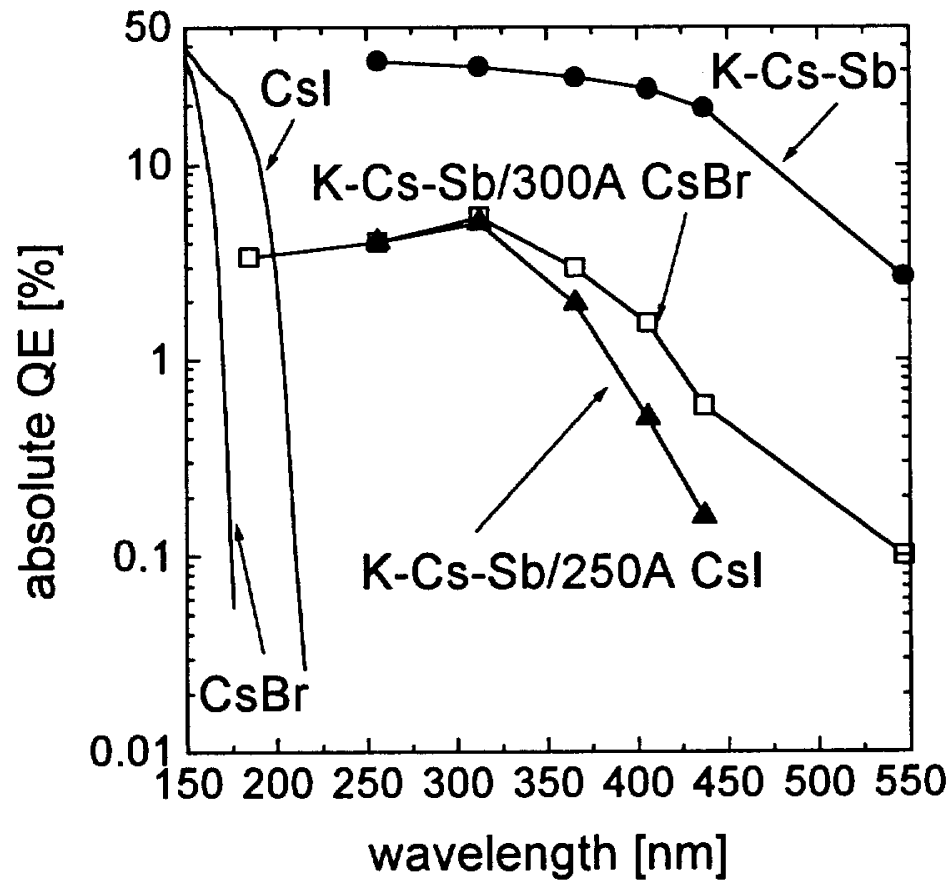

Figure 2 


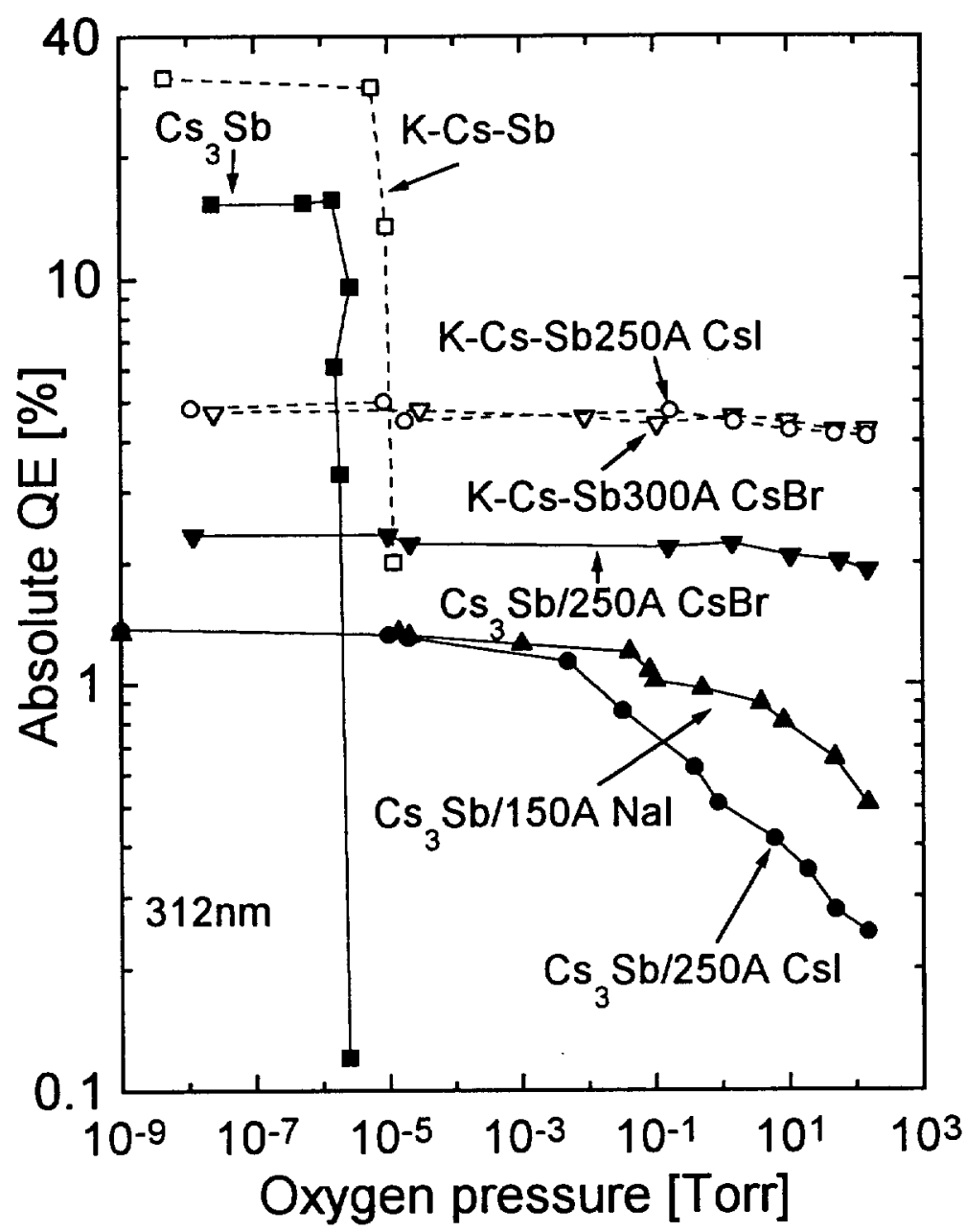

Figure 3

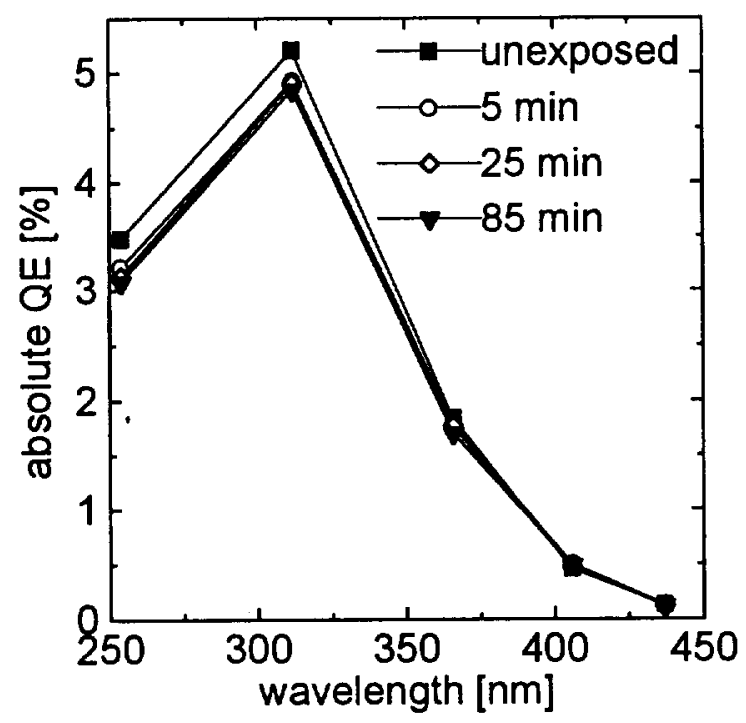

Figure 4 


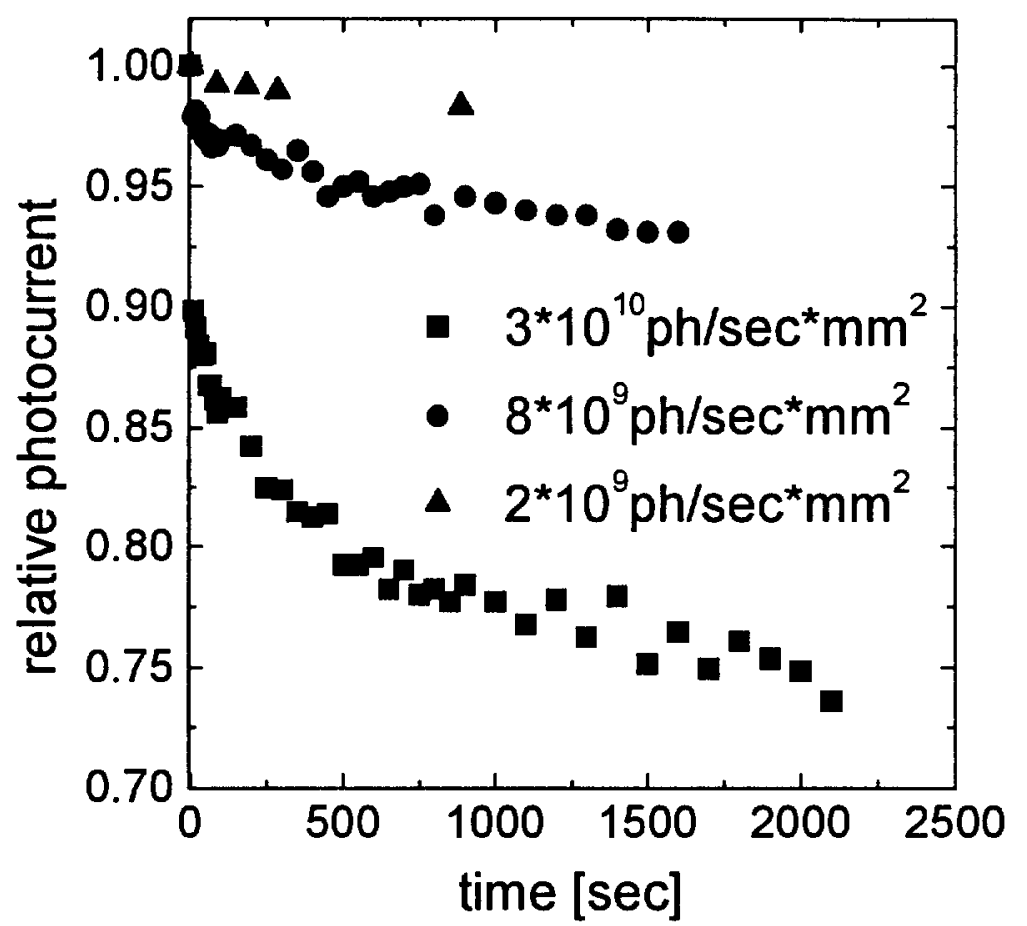

Figure 5

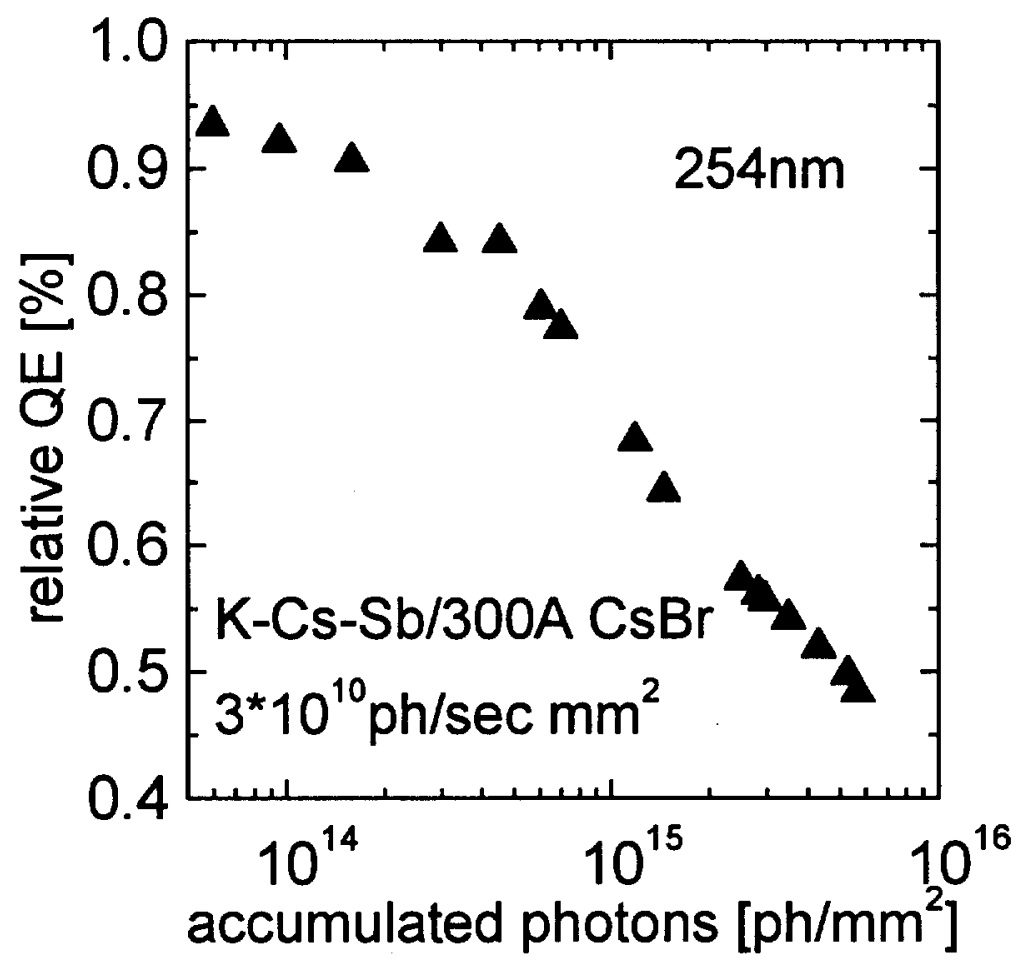

Figure 6

8 